\title{
SIRT1 promotes endometrial tumor growth by targeting SREBP1 and lipogenesis
}

\author{
LI LIN $^{1,2}$, XIAOXIA ZHENG ${ }^{3}$, CHUNPING QIU $^{1}$, SAMINA DONGOL $^{1}$, QINGTAO LV $^{4}$, \\ JIE JIANG ${ }^{1}$, BEIHUA KONG ${ }^{1}$ and CHENGUANG WANG ${ }^{5}$
}

\author{
${ }^{1}$ Department of Obstetrics and Gynecology, Qilu Hospital, Shandong University, Jinan; \\ ${ }^{2}$ Department of Obstetrics and Gynecology, Liaocheng People's Hospital, Liaocheng; \\ ${ }^{3}$ Department of Obstetrics and Gynecology, Jinan Maternity and Child Care Hospital, Jinan; \\ ${ }^{4}$ Department of Pharmaceutical Chemistry, Shandong University of Traditional Chinese Medicine, Jinan, \\ Shandong, P.R. China; ${ }^{5}$ Departments of Cancer Biology, Stem Cell Biology and Regenerative Medicine, \\ Kimmel Cancer Center, Thomas Jefferson University, Philadelphia, PA, USA
}

Received June 25, 2014; Accepted August 20, 2014

DOI: 10.3892/or.2014.3521

\begin{abstract}
Silent information regulator 1 (SIRT1) is involved in a number of cellular regulatory mechanisms affecting cellular life span, stress resistance, apoptosis and cellular metabolism. Recent studies have revealed that SIRT1 plays a dual role as a tumor suppressor and a tumor promoter in multiple stages of carcinogenesis. Increased lipogenesis has been found in cancer cells, sterol regulatory element binding protein 1 (SREBP1) are nuclear lipogenic transcription factors, which mainly regulate lipogenic processes by activating genes involved in fatty acid and triglyceride biosynthesis. In the present study, we detected expression of SIRT1 in endometrial cancer (EC) and illustrated the relationship between SIRT1 and SREBP1, which indicated that SIRT1 could stimulate endometrial tumor growth through the lipogenic pathway. Gene expression levels of SIRT1 were assayed using quantitative real-time PCR and protein expression levels were detected by western blotting. RNA interference was conducted in order to explore the subsequent effect on tumor cells and on the expression of SREBP1. Expression levels of SIRT1 in EC were found to be significantly higher than in normal endometrium. Knockdown of SIRT1 could downregulate expression of SREBP1 and suppress cell proliferation. These results demonstrated that SIRT1 may play a role as a tumor promoter in EC and can promote endometrial tumor growth by promoting lipogenesis. Our findings suggest that targeting SIRT1 may provide a theoretical basis for the management of EC.
\end{abstract}

Correspondence to: Dr Jie Jiang, Department of Obstetrics and Gynecology, Qilu Hospital, Shandong University, 107 Wenhuaxi Road, Jinan, Shandong 250012, P.R. China

E-mail: qljiangjie@sdu.edu.cn

Key words: SIRT1, SREBP1, endometrial cancer

\section{Introduction}

Endometrial cancer (EC) is the most common malignancy of the female reproductive tract and its incidence is on the increase (1). Approximately $40 \%$ of all cases can be attributed to obesity (2). Aberration in lipid metabolism contributes to different aspects of tumorigenesis (3); our research team focused on this domain of EC in recent years.

NAD-dependent class III histone deacetylase silent information regulator 1 (SIRT1), that shares the highest degree of homology with the yeast protein SIR2, can deacetylate both histones and non-histone proteins (4). Its deacetylation activity enables it to interact with a variety of important transcription factors and transcriptional co-regulatory factors, to regulate gene transcription, chromosome stability and activity of target proteins, which are involved in tumor metabolism and development $(5,6)$. The current results demonstrated that SIRT1 plays a dual role as a tumor promoter as well as a tumor suppressor (7). Its involvement in tumorigenesis may be due to its diverse distribution in different tissues and different upstream and downstream regulatory factors that regulate its function (8).

Sterol regulatory element binding protein 1 (SREBP1) belongs to the family of the basic helix-loop-helix leucine zipper family of DNA binding transcription factors, which can regulate most enzymes involved in fatty acid biosynthesis, such as acetyl-CoA carboxylase, fatty acid synthase, Elovl-6 and stearoyl-CoA desaturase (9). Lipogenesis is increased in cancer cells $(10,11)$, and the expression of SREBP1 has been observed to be elevated in various types of cancer (12-15). In our previous studies, we demonstrated that SREBP1 is overexpressed in endometrial and ovarian cancer and the expression of SREBP1 protein increased with higher FIGO surgical stage and histological grade of the disease. Furthermore, knockdown of SREBP1 could induce apoptosis, reduce cell proliferation in vitro and in vivo $(16,17)$. Eberhard et al demonstrated that inhibition of SREBP1 or its downstream target fatty acid synthase sensitized resistant cells to death ligands (18). 

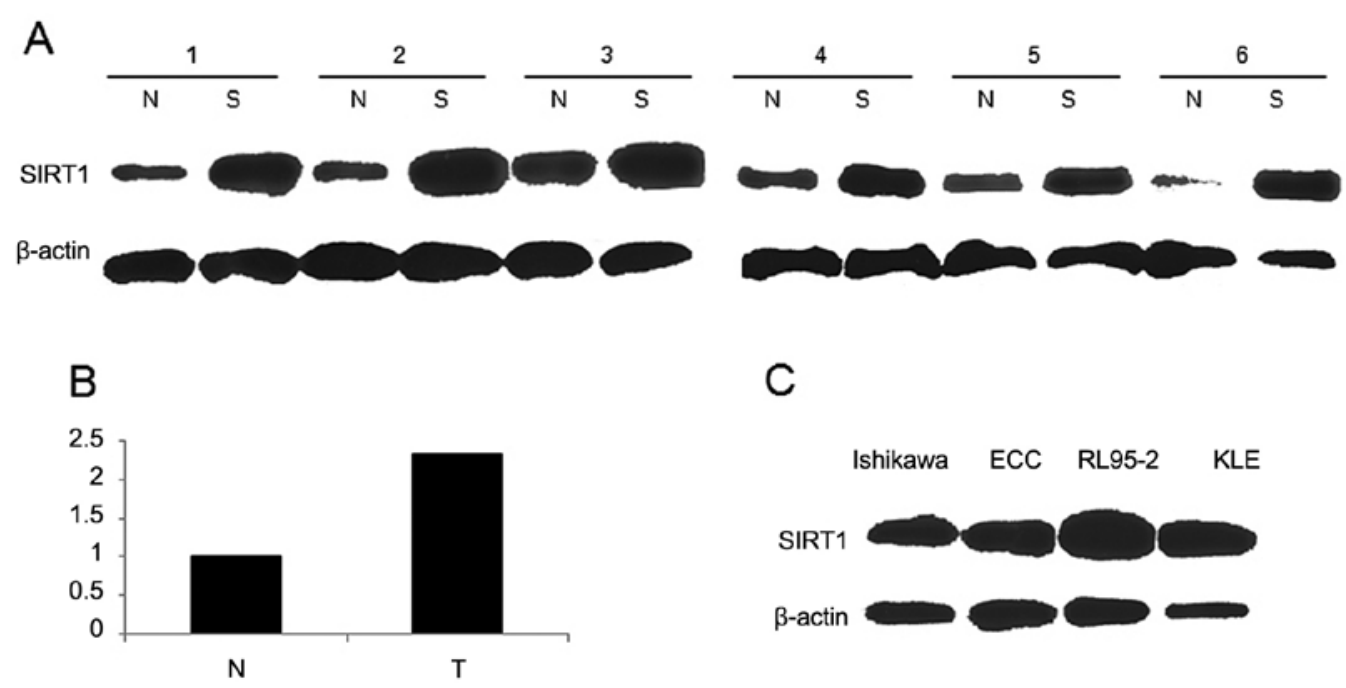

Figure 1. (A) Western blot analysis of SIRT1 expression in 6 selected human EC tissues (T) paired with adjacent normal endometrium tissues (N), T compared with N, P $<0.05$. (B) The relative expression of SIRT1 mRNAs illustrated by histogram. SIRT1 expression was higher in EC tissues, compared with adjacent endometrium tissues, $\mathrm{P}<0.05$. (C) Western blot analysis of SIRT1 expression in EC cell lines, the relatively higher expression in RL95-2 cell line. SIRT1, silent information regulator 1 ; EC, endometrial cancer.

Therefore, SREBP1 plays a role as a cancer promoter in human cancers.

In the present study, we investigated SIRT1 expression in EC and its effect on tumor cells. We also explored the correlation between SIRT1 and SREBP1 and we propose to establish the role of SIRT1 in EC.

\section{Materials and methods}

Tissue collection and cell culture. Sixty cases of fresh uterine endometrial adenocarcinoma and adjacent normal endometrial tissues were obtained from patients who underwent initial hysterectomy. All specimens were preserved at $-80^{\circ} \mathrm{C}$ refrigeration. Endorsed informed consent from all patients was collected prior to the operation. Human EC cell lines Ishikawa, ECC, RL95-2, KLE were separately cultured in RPMI-1640, MEM, DMEM/F12, DMEM/F12-medium. The basal medium was supplemented with $10 \%$ FBS. All cells were cultured at $37^{\circ} \mathrm{C}$ in a humid atmosphere with $5 \% \mathrm{CO}_{2}$.

Quantitative real-time PCR analysis. Total RNA was extracted from collected tissues and cultured cells by TRIzol reagent following the manufacturer's protocol (Invitrogen). Complementary DNAs were synthesized from total RNA using the SuperScript ${ }^{\mathrm{TM}}$ II Reverse Transcriptase kit (Invitrogen). PCR was operated using SYBR-Green Real-Time PCR Master Mix (Invitrogen). GAPDH was used as a control of normalization, the primers used for qRT-PCR are listed below. The gene symbol, forward primer sequence and reverse primer sequence (amplification size) were: SIRT1, 5'-TGT GAA AGT GAT GAG GAG GAT AGA and 5'-TAC AGC AAG GCG AGC ATA AAT A (136 bp); SREBP1a, 5'-CGG CGC TGC TGA CCG ACA TC and 5'-CCC TGC CCC ACT CCC AGC AT (104 bp); SREBP1c, 5'-GCG CAG ATC GCG GAG CCA T and 5'-CCC TGC CCC ACT CCC AGC AT (116 bp); GAPDH, 5'-GAG TCA ACG GAT TTG GTC GT and 5'-TTG AGG TCA ATG AAG GGG TC (103 bp).
Western blot analysis. The collected tissues and cultured cells were lysed using cell lysis buffer. Protein concentrations were determined by the BCA protein quantitative analysis kit. The dissolved lysates were subjected to $10 \%$ SDS-PAGE and blotted onto a PVDF membrane. After blocking for $2 \mathrm{~h}$ at room temperature, the membrane was incubated with primary antibodies against SIRT1 and SREBP1 (Santa Cruz Biotechnology, Inc.) at $4^{\circ} \mathrm{C}$ overnight. Horseradish peroxidase-labeled secondary antibody was used to bind the primary antibody. Proteins were visualized by ECL plus system. $\beta$-actin was used as a housekeeping protein. Quantitative data were analyzed using Bio-Rad software.

Cell transduction. Lentiviral vectors containing SIRT1 siRNAs and their controls were constructed and prepared for virus solutions by Genesil (Wuhan, China), and these solutions were then utilized to infect EC cell lines. The efficiency of transduction was determined by western blotting.

Plate colony formation assay. Logarithmic growth phase cells $(2,000)$ were seeded in $100-\mathrm{mm}$ culture plates for two weeks. Each group was allotted three plates. Colonies formed of each plate were stained with crystal violet, and statistics on the number of colonies were determined by Gel-Pro Analyzer.

Cell proliferation assays. Stably transfected cells were seeded at a density of $2 \times 10^{5}$ cells in 60 -mm culture plates. Each group had three plates. The total number of cells was counted for 6 days. Experiments were repeated three times.

Statistical analysis. Relative quantification of RNA expression was measured by using the $2^{-\Delta \Delta C t}$ method. Comparison of the quantitative data was analyzed by Student's t-test. The data was described as means $\pm \mathrm{SD}$. The statistical analyses were performed using SPSS 13.0 software (SPSS Inc.). p-values $<0.05$ were considered to indicate a statistically significant difference. 
A

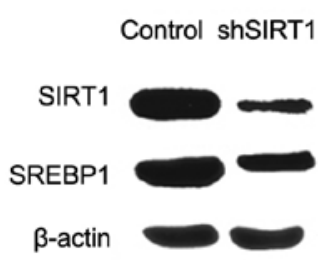

B

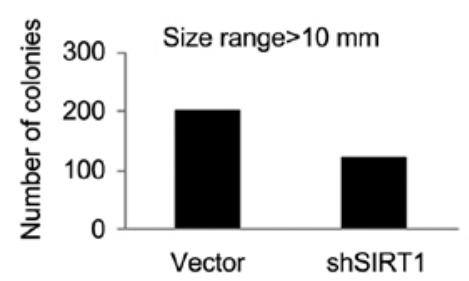

C

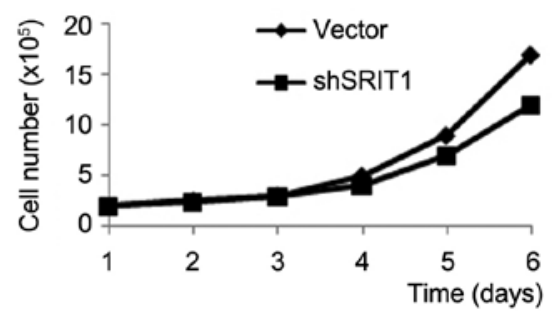

Figure 2. (A) Western blot analysis of a successful knockdown of SIRT1 and reduced expression of SREBP1 in transduced RL95-2 cell lines, P $<0.001$, P $<0.05$, compared with the control-shRNA group. (B) Curves of cell growth drawn by counting the cells at different time points as indicated. Cell growth capacity was lower in transduced RL95-2 cells compared with the control-shRNA group, $\mathrm{P}<0.05$. (C) The result of colony formation experiments showed that the colony numbers with knockdown of SIRT1 were relatively reduced compared with the control-shRNA group, P<0.05. SIRT1, silent information regulator 1; SREBP1, sterol regulatory element binding protein 1 .

\section{Results}

SIRT1 is upregulated in EC. To detect the expression of SIRT1 in EC, we analyzed the SIRT1 mRNA and protein levels by RT-PCR and western blotting in 40 cases of uterine endometrial adenocarcinoma and adjacent normal endometrial tissues. Compared to the normal controls, the expression of SIRT1 mRNA in EC was significantly elevated (Fig. 1A), the expression at protein levels showed the same trend, consistent with mRNA levels (Fig. 1B). These data demonstrated that SIRT1 is upregulated in EC. We also detected protein levels in four EC cell lines (Fig. 1C). RL95-2 cell line that had higher expression of SIRT1 was transduced with lentiviral vectors in order to knock down the expression of SIRT1 in the following study.

Effect on SREBP1 with the silencing of SIRT1 in RL95-2 cell line. To explore the correlation between SIRT1 and SREBP1 in EC, we used the lentiviral vectors with SIRT1 siRNAs in RL95-2 cell line. Efficiency of transduction was detected by western blotting (Fig. 2A). The expression of SREBP1 was clearly reduced at the protein levels (Fig. 2A); lowered expression of SIRT1 downregulated the expression of SREBP1. Accordingly, we established that SIRT1 positively regulates SREBP1 in EC. Our previous study showed that overexpression of SREBP1 was found in EC and it promoted tumor cell proliferation and tumor growth (16), and since the inhibition of SIRT1 can lower the expression of SREBP1, it can suppress tumor growth and progression. We further studied the role of SIRT1 in tumor growth in the following experiment.

Knockdown of SIRT1 inhibits EC cell growth capacity. To illustrate the effect of SIRT1 knockdown on cell growth capacity, we carried out direct cell counting and colony-forming assays. We found that cell number and the number of colonies were clearly reduced in the RL95-2 cell line with silencing of SIRT1 compared to the vector control (Fig. $2 \mathrm{~B}$ and C). These results demonstrate that SIRT1 plays a significant role in EC cell growth.

\section{Discussion}

Accumulating evidence indicates that SIRT1 plays a dual role in tumorigenesis. Elevated expression of SIRT1 has been reported in some types of human cancer tissues, such as pros- tate, liver, breast and stomach (19-22). Moreover, Zhao et al discovered that SIRT1 RNAi knockdown induces apoptosis and senescence, inhibits invasion and enhances chemosensitivity in pancreatic cancer cells (23). Nakane et al found that inhibition of cortactin and SIRT1 expression attenuates migration and invasion of prostate cancer DU145 cells (24). However, other studies demonstrated that SIRT1 is downregulated in several tumors $(25,26)$. There is little research exploring the correlation that exists between SIRT1 and EC.

We showed that SIRT1 is overexpressed in EC compared to normal endometrium, and its expression varies in a few EC cell lines. We also discovered that knockdown of SIRT1 clearly suppressed cell proliferation and cell growth. These results demonstrate that SIRT1 acts as a tumor promoter in EC. Knockdown of SIRT1 can affect biological behavior, yet it is insufficient to inhibit tumor progression. This suggests that SIRT1 alone may not be adequate in the promotion of carcinogenesis; however, downregulation of SIRT1 is clearly beneficial for EC treatment.

Many studies have shown an increased degree of lipogenesis in cancer cells and that the majority of fatty acids in cancer cells are derived from de novo fatty acid synthesis, in contrast to normal cells that obtain their fatty acids from the circulation $(3,10,27)$. SIRT1 acts as a sensor and a regulator in the metabolism of cancer (28). SREBP1 is the pivotal transcription factor in lipogenesis (29), and Bengoechea-Alonso et al speculated that SREBP1 may provide a link between lipid synthesis, and cell growth and proliferation (30). Based on these findings, some researchers have found that SREBP1 plays a role as an oncogene in human cancer (12-15). It has been reported that SIRT1 deacetylates and inhibits SREBP-1c transactivation by decreasing its stability and its occupancy at the lipogenic genes. Furthermore, hepatic overexpression of SIRT1 or treatment with resveratrol can decrease elevated acetylated SREBP-1c levels in diet-induced obese mice (31). Walker et al also demonstrated that SIRT1 negatively regulates SREBP1 during fasting, and decreased expression of SREBP1 correlates with decreased hepatic lipid and cholesterol levels and attenuates liver steatosis in diet-induced and genetically obese mice (32). In contrast, Defour discovered that knocking out the catalytic domain of SIRT1 decreased SREBP-1c mRNA and protein levels, and SREBP-1c promoter transactivation was significantly increased in response to SIRT1 overexpression by in vivo genetic electrotransfer in skeletal muscle (33). The 
variance in the contribution of SIRT1 in regulating SREBP1 negatively or positively depends on genetic background, tissue types and metabolic environment. The correlation of SIRT1 and SREBP1 in cancer tissues has not been found.

Our previous studies showed that SREBP1 plays a role as a tumor promoter in EC; in the present study, we established the role of SIRT1 as a cancer promoter in EC. The expression of SREBP1 was downregulated by transduction of SIRT1-specific siRNA lentivirus in RL95-2 cell line. Briefly, SIRT1 affects biological behaviors by targeting SREBP1 and lipogenesis. Activated FoxO1 inhibits SREBP-1c gene expression by reducing transcriptional activity of Sp1 and SREBP-1c and disrupting the assembly of transcriptional initiation complex on the SREBP-1c promoter $(34,35)$. In cancer, SIRT1 suppresses Fox01 activity by deacetylating (36-38), while low expression of Fox01 decreases suppression of SREBP1, consequently resulting in elevated levels of SREBP1. However, the exact mechanism remains unclear and will be studied further in subsequent analyses.

Obesity is an established epidemiological risk factor in EC (2). On the one hand, obese patients have increased endogenous estrogen levels, which comes from conversion of androstenedione to estrone and the aromatization of androgens to estradiol in redundant subcutaneous adipocytes; on the other hand, abnormal lipometabolism exists in obese patients and disorder of lipid metabolism contributes to different aspects of tumorigenesis. In the present study, SIRT1 was demonstrated to promote endometrial tumor growth by increasing the levels of SREBP1 and lipogenesis, thus we postulate that we can attenuate tumor growth by targeting SIRT1 in EC.

Collectively, we confirmed that SIRT1 significantly upregulates and can promote tumor proliferation, migration and invasion capacity by targeting SREBP1 and lipogenesis in EC. Thus, knockdown of SIRT1 or SIRT1 inhibitor may play a substantial role in suppressing endometrial tumor. Thus, SIRT1 may be regarded as a therapeutic target in EC.

\section{Acknowledgements}

This study was partly funded by the National Natural Science Foundation of China [81372808 (J.J.)] and [81173614 (Q.T.L)], and was also partly funded by the Science, and the Science and Technology Developing Planning of Jinan (201303035), and the Technology Development planning of Shandong [2012G0021823 (J.J)].

\section{References}

1. Siegel R, Naishadham D and Jemal A: Cancer statistics, 2013. CA Cancer J Clin 63: 11-30, 2013.

2. Modesitt SC, Hsu JY, Chowbina SR, Lawrence RT and Hoehn KL: Not all fat is equal: differential gene expression and potential therapeutic targets in subcutaneous adipose, visceral adipose, and endometrium of obese women with and without endometrial cancer. Int J Gycecol Cancer 22: 732-741, 2012.

3. Santos CR and Schulze A: Lipid metabolism in cancer. FEBS J 279: 2610-2623, 2012.

4. Imai S: The molecular mechanism of aging and longevity and the function of Sir2 proteins. Nihon Ronen Igakkai Zasshi 38: 735-739, 2001 (In Japanese).

5. Liu T, Liu PY and Marshall GM: The critical role of the class III histone deacetylase SIRT1 in cancer. Cancer Res 69: 1702-1705, 2009.
6. Katto J, Engel N, Abbas W, Herbein G and Mahlknecht U: Transcription factor $\mathrm{NF} \kappa \mathrm{B}$ regulates the expression of the histone deacetylase SIRT1. Clin Epigenetics 5: 11, 2013.

7. Song NY and Surh YJ: Janus-faced role of SIRT1 in tumorigenesis. Ann NY Acad Sci 1271: 10-19, 2012.

8. Fang Y and Nicholl MB: Sirtuin 1 in malignant transformation: friend or foe? Cancer Lett 306: 10-14, 2011.

9. Shimano H: SREBPs: physiology and pathophysiology of the SREBP family. FEBS J 276: 616-621, 2009.

10. Swinnen JV, Brusselmans K and Verhoeven G: Increased lipogenesis in cancer cells: new players, novel targets. Curr Opin Clin Nutr Metab Care 9: 358-365, 2006.

11. Menendez JA and Lupu R: Fatty acid synthase and the lipogenic phenotype in cancer pathogenesis. Nat Rev Cancer 7: 763-777, 2007.

12. Li JN, Mahmoud MA, Han WF, Ripple M and Pizer ES: Sterol regulatory element-binding protein-1 participates in the regulation of fatty acid synthase expression in colorectal neoplasia. Exp Cell Res 261: 159-165, 2000.

13. Swinnen JV: Increased lipogenesis in steroid-responsive cancer cells: mechanisms of regulation, role in cancer cell biology and perspectives on clinical applications. Verh K Acad Geneeskd Belg 63: 321-333, 2001.

14. Yang Yu, Morin PJ, Han WF, et al: Regulation of fatty acid synthase expression in breast cancer by sterol regulatory element binding protein-1c. Exp Cell Res 282: 132-137, 2003.

15. Yahagi N, Shimano H, Hasegawa K, et al: Co-ordinate activation of lipogenic enzymes in hepatocellular carcinoma. Eur J Cancer 41: 1316-1322, 2005.

16. Li W, Tai Y, Zhou J, et al: Repression of endometrial tumor growth by targeting SREBP1 and lipogenesis. Cell Cycle 11: 2348-2358, 2012.

17. Nie LY, Lu QT, Li WH, et al: Sterol regulatory element-binding protein 1 is required for ovarian tumor growth. Oncol Rep 30: 1346-1354, 2013.

18. Eberhard Y, Gronda M, Hurren R, et al: Inhibition of SREBP1 sensitizes cells to death ligands. Oncotarget 2: 186-196, 2011.

19. Huffman DM, Grizzle WE, Bamman MM, et al: SIRT1 is significantly elevated in mouse and human prostate cancer. Cancer Res 67: 6612-6618, 2007.

20. Choi HN, Bae JS, Jamiyandorj U, et al: Expression and role of SIRT1 in hepatocellular carcinoma. Oncol Rep 26: 503-510, 2011.

21. Kalle AM, Mallika A, Badiger J, Alinakhi and Talukdar P and Sachchidanand: Inhibition of SIRT1 by a small molecule induces apoptosis in breast cancer cells. Biochem Biophys Res Commun 401: 13-19, 2010.

22. Cha EJ, Noh SJ, Kwon KS, et al: Expression of DBC1 and SIRT1 is associated with poor prognosis of gastric carcinoma. Clin Cancer Res 15: 4453-4459, 2009.

23. Zhao G, Cui J, Zhang JG, et al: SIRT1 RNAi knockdown induces apoptosis and senescence, inhibits invasion and enhances chemosensitivity in pancreatic cancer cells. Gene Ther 18: 920-928, 2011.

24. Nakane K, Fujita Y, Terazawa R, et al: Inhibition of cortactin and SIRT1 expression attenuates migration and invasion of prostate cancer DU145 cells. Int J Urol 19: 71-79, 2012.

25. Firestein R, Blander G, Michan S, et al: The SIRT1 deacetylase suppresses intestinal tumorigenesis and colon cancer growth. PLoS One 3: e2020, 2008.

26. Lai CC, Lin PM, Lin SF, et al: Altered expression of SIRT gene family in head and neck squamous cell carcinoma. Tumour Biol 34: 1847-1854, 2013.

27. Medes G, Thomas A and Weinhouse S: Metabolism of neoplastic tissue. IV. A study of lipid synthesis in neoplastic tissue slices in vitro. Cancer Res 13: 27-29, 1953.

28. Knight JR and Milner J: SIRT1, metabolism and cancer. Curr Opin Oncol 24: 68-75, 2012.

29. Eberlé D, Hegarty B, Bossard P, Ferré P and Foufelle F: SREBP transcription factors: master regulators of lipid homeostasis. Biochimie 86: 839-848, 2004.

30. Bengoechea-Alonso MT, Punga T and Ericsson J: Hyperphosphorylation regulates the activity of SREBP1 during mitosis. Proc Natl Acad Sci USA 102: 11681-11686, 2005.

31. Ponugoti B, Kim DH, Xiao Z, et al: SIRT1 deacetylates and inhibits SREBP-1C activity in regulation of hepatic lipid metabolism. J Biol Chem 285: 33959-33970, 2010.

32. Walker AK, Yang F, Jiang K, et al: Conserved role of SIRT1 orthologs in fasting-dependent inhibition of the lipid/cholesterol regulator SREBP. Genes Dev 24: 1403-1417, 2010. 
33. Defour A, Dessalle K, Castro Perez A, et al: Sirtuin 1 regulates SREBP-1c expression in a LXR-dependent manner in skeletal muscle. PLoS One 7: e43490, 2012.

34. Deng X, Zhang W, O-Sullivan I, et al: FoxO1 inhibits sterol regulatory element-binding protein-1c (SREBP-1c) gene expression via transcription factors Sp1 and SREBP-1c. J Biol Chem 287: 20132-20143, 2012.

35. Zhang W, Patil S, Chauhan B, et al: FoxO1 regulates multiple metabolic pathways in the liver: effects on gluconeogenic glycolytic, and lipogenic gene expression. J Biol Chem 281: 10105-10117, 2006.
36. Motta MC, Divecha N, Lemieux M, et al: Mammalian SIRT1 represses forkhead transcription factors. Cell 116: 551-563, 2004.

37. Yang $Y$, Hou H, Haller EM, Nicosia SV and Bai W: Suppression of FOXO1 activity by FHL2 through SIRT1mediated deacetylation. EMBO J 24: 1021-1032, 2005.

38. Frampton G, Ueno Y, Quinn M, et al: The novel growth factor, progranulin, stimulates mouse cholangiocyte proliferation via sirtuin-1-mediated inactivation of FOXO1. Am J Physiol Gastrointest Liver Physiol 303: G1202-G1211, 2012. 